\title{
Theoretical study of spectroscopic data of a dihidrochalcone from the natural product metrodorea
}

\section{Renato Araújo da Costa ${ }^{1,2}$, Fabiana Cristina de Araújo Nascimentoㅇ, Sebastião Gomes Silva $^{1}$, Andréia do Socorro Sousa Silva ${ }^{1}$ e Davi do Socorro Barros Brasil ${ }^{1}$}

\author{
${ }^{1}$ Universidade Federal do Pará. Rua Augusto Corrêa, 01 - Guamá. CEP 66075-110 - Belém Pará - Brasil. \\ ${ }^{2}$ Instituto Federal do Pará. Rodovia PA 275, s/n - União. CEP 68515-000 - Parauapebas- Pará - Brasil.
}

Email: renatoac@ufpa.br,renato.costa@ifpa.edu.br

Received: January $27^{\text {th }}, 2017$

Accepted: February $17^{\text {th }}, 2017$

Published: Mach 30 3017

Copyright (C2016 by authors and Institute of Technology Galileo of Amazon (ITEGAM). This work is licensed under the Creative Commons Attribution International License (CC BY 4.0).

http://creativecommons.org/licenses/by/4.0/

\begin{abstract}
The DFT quantum method has become a useful computational tool to aid in the interpretation of experimental data from natural NMR products. Recently, a dihydrochalcone: 1- (5,7-dihydroxy-2,2dimethylchroman-6-yl) -3- (2,2-dimethylchroman-6-yl) propan-1-one (1) was isolated from species Metrodorea stipularis. The computational method DFT/B3LYP/cc-pVDZ was used to optimize the structures the chemical structure of dihydrochalcone and to calculate chemical shifts $(\delta \mathrm{H}$ and $\delta \mathrm{C}) . \delta \mathrm{H}$ and $\delta \mathrm{C}$ were calculated and compared with the experimental data. Comparison of the NMR data by simple linear regression (SLR) showed satisfactory statistical results with a correlation coefficient (R2) and predictive ability (Q2) of over 97\% thus demonstrating the efficiency of the method used.
\end{abstract}

Keywords: Dihidrochalcona; NMR; DFT.

\begin{abstract}
RESUMO
O metódo quântico de DFT tem se tornado uma ferramenta computacional útil para ajudar na interpretação de dados experimentais de produtos naturais via RMN. Recentemente, foi isolados uma dihidrochalcona: 1-(5,7-dihidroxi-2,2-dimetilcroman-6-il) -3- (2,2-dimetilcroman-6-il) propan-1-ona (1) a partir a especie Metrodorea stipularis. O método computacional DFT/B3LYP/cc-pvDZ foi aplicado para otimizar a estrutra química da dihidrochalcona e para os cálculos de deslocamentos químicos $(\delta \mathrm{H}$ e $\delta \mathrm{C})$. Os $\delta \mathrm{H}$ e $\delta \mathrm{C}$ foram calculados e comparados com os experimentais. A comparação dos dados de RMN pela regressão linear simples mostrou resultados estatísticos satisfatórios com coeficiente de correlação (R2) e capacidade de previsão (Q2) superior a 98\% demostrando, com isso, a eficiência do método empregado.
\end{abstract}

Palavras Chaves: Dihidrochalcona; RMN; DFT.

\section{INTRODUÇÃO}

A espectroscopia de ressonância magnética nuclear (RMN) é uma das técnicas experimentais mais poderosas para a obtenção de estruturas tridimensionais de moléculas complexas, principalmente para a análise das configurações relativas e absolutas de compostos orgânicos [1] Devido à versatilidade das técnicas de RMN, bem como a quantidade de informação que pode ser extraída da análise de espectros de RMN, essa informação é extremamente importante na química dos produtos naturais onde esta ferramenta é mais aplicável [2].

Adicionado às tecnicas de RMN, cálculos da mecânica quântica tem se tornado uma ferramentas computacionais útil para 
ajudar na interpretação dos dados experimentais espectroscópicos de produtos naturais via RMN [1][2][3].

Vários artigos forma publicados [1-6] aplicando a modelagem molecular em seus trabalhos como uma alternativa promissora em auxílio às técnicas experimentais tradicionais de RMN alcançando resultados precisos em um tempo de obtenção de dados significativamente pequeno. Pode-se se afirmar então que, atualmente, a simulação computacional é um ramo da ciência tão importante quanto as técnicas experimentais [3].

Neste sentido, o presente estudo, utiliza métodos teóricos quânticos, a nível de DFT, para determinação de dados estruturais (geométricos) e de deslocamentos quimicos $(\delta \mathrm{H}$ e $\delta \mathrm{C})$ de uma dihidrochalcona: 1-(5,7-dihidroxi-2,2-dimetilcroman-6-il) -3(2,2-dimetilcroman-6-il) propan-1-ona (figura 1), isolada apartir a especie Metrodorea stipularis [7]. Os valores teóricos dos $\delta \mathrm{H}$ e $\delta \mathrm{C}$ obtidos foram comparados aos experementais via regressão linear simples, afim de verificar o grau correlação, significância e previsibilidade dos modelos obtidos e com isso mostar a eficiencia da motodoligia empregada no estudo.

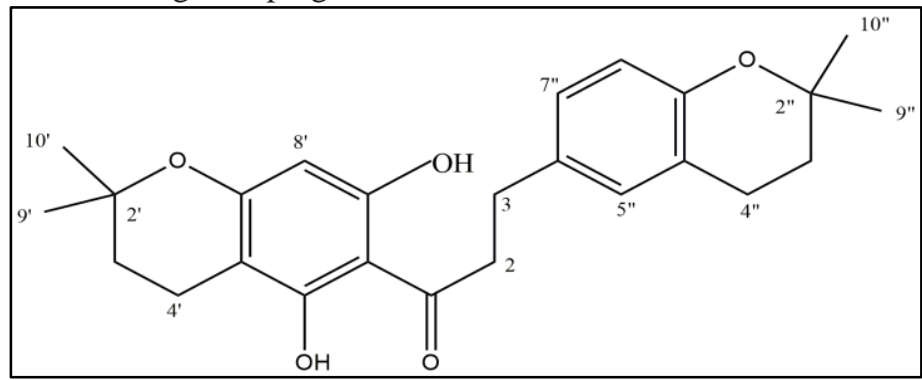

Figura 1 Estrutura da dihidrochalcona.

Fonte: Autores (2016).

\section{MATERIAIS E MÉTODOS}

a. Método computacional

Inicialmente a dihidrochalcona (figura 1) foi desenhada e pré-otimizda pelo método de Mecânica Molecular PM3[8] utilizando o programa HyperChem Professional 7.5 [9]. Posteriomente para a otmização estrutura da geometria, cálculo de frequencia (para detrminação do ponto estacionário) e obtenção de dados de deslocamentos químicos de ${ }^{13} \mathrm{C} \mathrm{e}{ }^{1} \mathrm{H}$ foi realizado calculos quânticos a nível DFT com o funcional híbrido B3LYP com aplicação do conjunto de funções bases cc-pVDZ utilizando o programa GAUSSIAN 09 [10] sob influência do solvente acetona modelado através do método PCM [11,12]. O tetrametilsilano (TMS), utilizado como padrão interno, foi calculado, também, sob influência do solvente cetona pelo método DFT/B3LYP/cc-pVDZ. Os valores obtidos para as constantes de blindagem teóricas, referentes aos núcleos de ${ }^{13} \mathrm{C}$ e ${ }^{1} \mathrm{H}$, foram de 192,5621 e 31,2969 ppm, respectivamente.

\section{b. Análise estatística}

O software MINITAB 14 [13] foi utilizado para a análise estatística os dados teóricos de RMN comparados aos experimentais via regressão linear. Os parâmetros estatísticos mais importantes como, coeficiente de correlação ou grau de ajuste $\left(R^{2}\right)$, desvio padrão $(S)$, graus de significância $(F)$ e previsibilidade $\left(Q^{2}\right.$ e SPRESS ) foram os escolhidos para esta analise. Os parâmetros estatísticos para o aprimoramento dos cálculos, tais como, coeficientes $\mathrm{a}$ e $\mathrm{b}$ da regressão linear $\delta$ calc $=\mathrm{a}+\mathrm{b} \delta$ exp; erro absoluto médio $(\mathrm{EAM})$ definido como $\mathrm{EAM}=\Sigma \mathrm{n}|\delta \mathrm{calc}-\delta \exp | / \mathrm{n}$, e erro absoluto médio corrigido $($ EAMC), definido por EAMC = $\Sigma \mathrm{n} \mid \delta$ corr $-\delta \exp \mid / \mathrm{n}$, onde $\delta$ corr $=(\delta$ calc $-\mathrm{a}) / \mathrm{b}[14]$ foram obtidos Estes parâmetros, calculados para dados experimentais e teóricos nessa estrutura, permite o estudo de deslocamentos químicos (ppm), bem como de resíduos: $\mathrm{RS}=\mid \delta \exp -\delta$ calc $\mid$ dos átomos de hidrogênio e de carbono.

As equações obtidas, pelo procedimento de regressão linear, foram testadas para seus poderes de ajuste, significância e previsibilidade usando um procedimento de validação cruzada Validação cruzada é um método prático e confiável para testes de significância. A chamada aproximação "leave-one-out" consiste em desenvolver uma série de modelos omitindo com uma amostra de cada vez. Após desenvolvimento de cada modelo, os dados omitidos são preditos e a diferença entre valores verdadeiros e valores preditos ( $\hat{y})$ são calculados. A soma dos quadrados destas diferenças é determinada e finalmente o desempenho do modelo (sua habilidade preditiva) pode ser dada por PRESS (soma do quadrado dos erros de predição) equação 1 e SPRESS (desvio padrão da validação cruzada) [14] equação 2 .

$$
\begin{aligned}
\text { PRESS } & =\sum_{i=1}^{n}\left(y_{i}-\hat{y}_{i}\right)^{2} \\
\text { PRPES } & \sqrt{\text { PR }}
\end{aligned}
$$

onde, y é o valor experimental, $\hat{y}$ é o valor predito, n é o número de amostras usadas para obter o modelo e $\mathrm{k}$ é o número de parâmetros de RMN.

A habilidade preditiva dos modelos foi também quantificada em termos de $\mathrm{Q}^{2}$, o qual é definido como [13]:

$$
Q=1.0 \frac{\sum_{i=1}^{n}\left(y_{i}-\hat{y}_{i}\right)^{2}}{\sum_{i=1}^{n}\left(y_{i}-\bar{y}_{i}\right)^{2}}
$$

onde $\bar{y}=y_{\text {medic }}$

Um modelo com elevado grau de previsibilidade apresentar $\mathrm{Q}^{2}$ próximo de 1 e $\mathrm{s}_{\text {press }}$ próximo de zero [14].

\section{RESULTADOS E DISCUSSÕES}

Os deslocamentos químicos experimentais e teóricos para RMN ${ }^{13} \mathrm{C} \mathrm{e}{ }^{1} \mathrm{H}$ da dihidrochalcona estão dispostos nas Tabelas $1 \mathrm{e}$ 2, bem como o resíduo (RS) em ppm para cada um dos átomos de carbono e átomos de hidrogénio presentes nas estruturas. A Tabela 1 mostra a proximidade existente entre os valores calculados dos deslocamentos químicos a nível de B3LYP/cc-pVDZ para a estrutura em estudo (Figura 1) e os valores experimentais, apresentando baixos valores residuais (diferença entre deslocamentos químicos experimentais e teóricos), o que confirma a eficiência do modelo computacional utilizado, mostrando que o método pode ser aplicado a substâncias similares, reforçando a ideia de que os métodos computacionais podem ser empregados em auxílio à interpretação de dados experimentais, fazendo com que tais métodos ganhem cada vez mais espaço no campo científico. 
Tabela 1. Dados de RMN ( $\delta \mathrm{H}$ e $\delta \mathrm{C})$ experimentais (Exp.), teóricos (DFT) e preditos (pelos modelos lineares) em ppm para a estrutura em estudo.

\begin{tabular}{|c|c|c|c|c|c|c|c|c|}
\hline \multirow[b]{2}{*}{ Posição } & \multicolumn{4}{|c|}{${ }^{13} \mathrm{C}$} & \multicolumn{4}{|c|}{${ }^{1} \mathrm{H}$} \\
\hline & Exp. & Teórico & Resíduo & Predito & Exp & $\begin{array}{c}\text { Teóric } \\
\text { o }\end{array}$ & Resíduo & $\begin{array}{c}\text { Predi } \\
\text { to }\end{array}$ \\
\hline C1 & 204,7 & 199,27 & 5,43 & 204,78 & & & & \\
\hline C2 & 45,5 & 54,24 & 7,74 & 9,92 & 3,30 & 3,52 & 0,22 & 3,78 \\
\hline C3 & 29,7 & 36,27 & 6,57 & 31,92 & 2,83 & & 0,20 & 2,86 \\
\hline$C 2^{\prime}$ & 75,6 & 79,25 & 3,65 & 77,50 & & & & \\
\hline C3' & 31,2 & 34,81 & 3,61 & 30,38 & 1,76 & 2,63 & 0,09 & 2,06 \\
\hline$C 4^{\prime}$ & 16,2 & 18,97 & 2,77 & 13,58 & 2,53 & 1,85 & 0,24 & 2,51 \\
\hline C4'a & 104,8 & 99,84 & 4,96 & 99,34 & & & & \\
\hline C5' & 157,0 & 156,49 & 0,51 & 159,42 & & & & \\
\hline$C 6^{\prime}$ & 100,0 & 108,56 & 8,56 & 108,59 & & & & \\
\hline$C 7^{\prime}$ & 162,2 & 155,29 & 6,91 & 158,14 & & & & \\
\hline C8' & 94,5 & 92,75 & 1,75 & 91,82 & 5,92 & 2,29 & 0,48 & 5,75 \\
\hline C8'a & 165,5 & 156,94 & 8,56 & 159,89 & & & & \\
\hline C9' & 25,5 & 31,47 & 5,97 & 26,84 & 1,22 & 5,44 & 0,26 & 1,68 \\
\hline C10' & 25,5 & 24,17 & 1,33 & 19,10 & 1,22 & 1,48 & 0,13 & 1,27 \\
\hline C2" & 73,2 & 77,87 & 4,67 & 76,04 & & & & \\
\hline C3" & 32,4 & 35,52 & 3,12 & 31,14 & 2,69 & 1,09 & 1,09 & 1,80 \\
\hline C4" & 22,1 & 25,80 & 3,70 & 20,82 & 2,69 & 1,60 & 0,41 & 2,50 \\
\hline C4"a & 120,0 & 121,85 & 1,85 & 122,68 & & & & \\
\hline C5" & 126,8 & 126,59 & 0,21 & 127,71 & 6,91 & 2,28 & 0,62 & 6,63 \\
\hline C6" & 132,2 & 136,46 & 1,26 & 134,99 & & & & \\
\hline C7"' & 128,1 & 129,18 & 1,08 & 130,45 & 6,93 & 6,29 & 0,06 & 7,22 \\
\hline C8" & 116,8 & 116,02 & 0,78 & 116,50 & 6,56 & 6,87 & 0,12 & 6,78 \\
\hline C8"a & 151,9 & 152,07 & 0,17 & 154,73 & & & & \\
\hline C9" & 25,5 & 24,51 & 0,99 & 19,45 & 1,33 & 0,85 & 0,48 & 1,03 \\
\hline C10" & 25,5 & 31,89 & 6,39 & 27,28 & 1,33 & 1,16 & 0,17 & 1,35 \\
\hline
\end{tabular}

Fonte: Autores (2016).

Na Figura 2 A e B, são mostrados os gráficos de correlação obtido após o tratamento por regressão linear dos dados teóricos e experimentais de RMN de ${ }^{13} \mathrm{Ce}^{1} \mathrm{H}$ da dihidrochalcona. Os parâmetros de maior relevância utilizados para análise estatística são mostrados na Tabela 2.
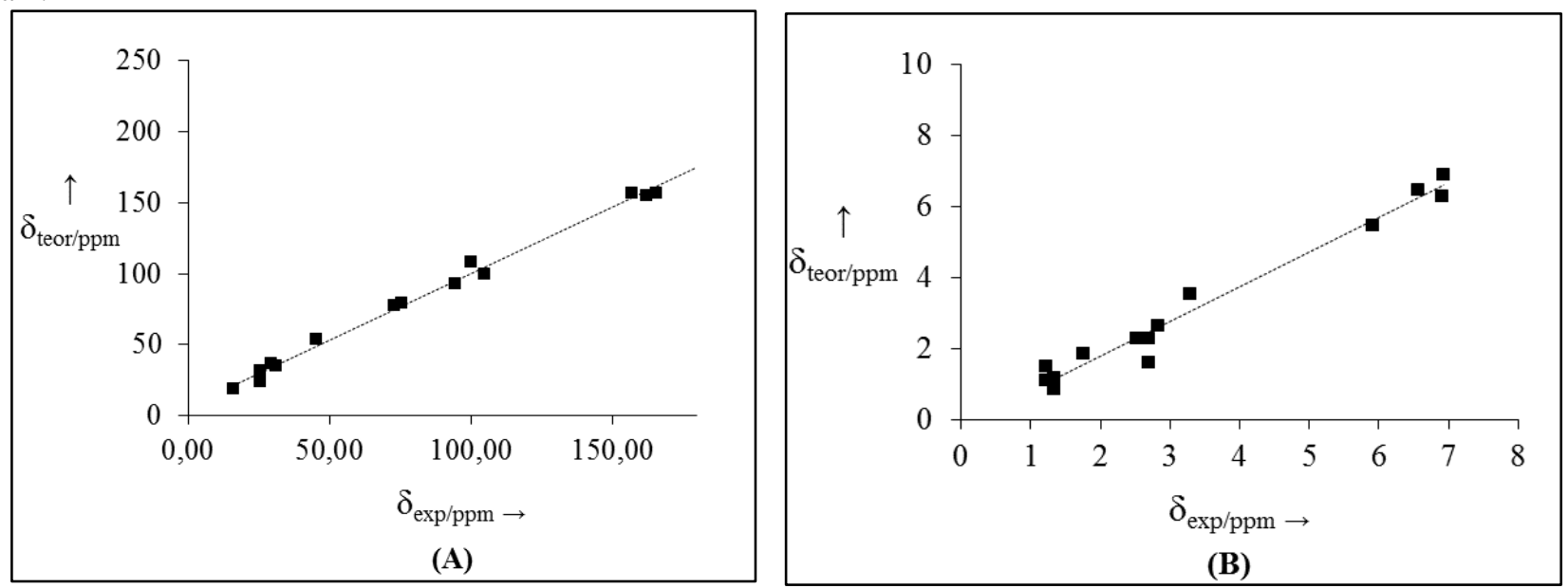

Figura 2: Gráficos de correlação entre os $\delta{ }^{13} \mathrm{C}(\mathrm{A})$ e ${ }^{1} \mathrm{H}$ (B) experimentais e calculados no pelo método teórico (B3LYP/cc-PVDZ) para a dihidrochalcona.

Fonte: Os Autores (2016). 
A análise dos dados estatísticos revela que o modelo de ${ }^{13} \mathrm{C}$ e ${ }^{1} \mathrm{H}$ calculado para a molécula em estudo apresenta uma boa correlação linear $\left(\mathrm{R}^{2}=99,6 \%\right.$ para ${ }^{13} \mathrm{C}$ e $\mathrm{R}^{2}=97,2 \%$ para $\left.{ }^{1} \mathrm{H}\right)$ e estão bem ajustados, além de apresentarem resultados sastifatorios e um alto grau de previsibilidade $(\mathrm{s}=3,51$; SPRESS $=0,79 ; \mathrm{F}=5714,82$; $\mathrm{Q}^{2}=99,53 \%$ para $\delta_{\mathrm{C}}$ e $\mathrm{s}=0,36 ;$ SPRESS $=0,12 ; \mathrm{F}=454,31 ; \mathrm{Q}^{2}=$ $96,65 \%$ para $\delta_{\mathrm{H}}$ ) como disposto na Tabela 2. Esta análise indica que há uma concordância linear entre os dados de deslocamento teóricos de ${ }^{13} \mathrm{C}$ e ${ }^{1} \mathrm{H}$ obtidos em relação aos valores experimentais para a substância estudada, pois são quantitativamente semelhantes aos deslocamentos citados na literatura, mostrando que o método teórico aplicado pode auxiliar de forma eficaz nas técnicas de Ressonância Magnética Nuclear.

Os valores de $R^{2}, F$ e $s$ mostram que os respectivos modelos possuem bons graus de ajuste e significância, bem como boa previsibilidade, verificado pelo valor de PRESS e $Q^{2}$. O tratamento estatístico baseado em regressão linear simples e procedimentos de validação cruzada reforça a proximidade existente entre os dados teóricos e calculados para a substancia estudada alcançando graus de correlação e previsibilidade $\left(\mathrm{Q}^{2}\right)$ de 99,53 para o ${ }^{13} \mathrm{C}$ e 96,65 para o ${ }^{1} \mathrm{H}$, além de bons graus de significância (F) (Tabela 3), gerando modelos lineares com boa representatividade estatística reforçando a eficácia do método teórico utilizado e mostrando que que essa metodologia pode ser aplicado ao estudo de produtos naturais similares a dihidrochalcona estudada nesse trabalho. Tabela 2. Parâmetros de ajuste linear das propriedades de RMN calculadas para a dihidrochalcona em estudo.

A Tabela 2: Gráficos de correlação entre os $\delta{ }^{13} \mathrm{C}(\mathrm{A})$ e ${ }^{1} \mathrm{H}$ (B) experimentais e calculados no pelo método teórico (B3LYP/ccPVDZ) para a dihidrochalcona.

\begin{tabular}{lll}
\hline Parâmetros & $\boldsymbol{\delta}^{\mathbf{1 3}} \mathbf{C}$ & $\boldsymbol{\delta}^{\mathbf{1}} \mathbf{H}$ \\
\hline $\mathbf{a}$ & 0,94 & 0,97 \\
$\mathbf{b}$ & 6,16 & $-0,15$ \\
EAM $^{[\mathbf{a}]}$ & 4,16 & 0,27 \\
EAMC $^{[\mathrm{a}]}$ & 3,02 & 0,22 \\
$\boldsymbol{S}_{\boldsymbol{R}^{\mathbf{2}}}$ & 3,51 & 0,36 \\
$\boldsymbol{Q}^{\mathbf{2}}$ & $99,6 \%$ & $97,2 \%$ \\
$\boldsymbol{F}$ & $99,53 \%$ & $96,65 \%$ \\
$\boldsymbol{P R E S S}^{[\mathbf{b}]}$ & 5714,82 & 454,31 \\
SPRESS $^{[\mathbf{b}]}$ & 331,925 & 2,075 \\
\hline
\end{tabular}

Fonte: Os Autores (2016).

\section{CONCLUSÃO}

Os deslocamentos químicos obtidos para os núcleos de ${ }^{1} \mathrm{H}$ e ${ }^{13} \mathrm{C}$ utilizando o método DFT B3LYP/cc-pVDZ são condizentes aos experimentais apresentando baixos resíduos, corroborando a utilização deste método em dihidrocalcona similares.

A análise de espectros de RMN nem sempre é uma tarefa fácil, contudo, técnicas de simulação computacionais têm ganhado espaço significativo no meio científico, oferecendo boas contribuições à Química Orgânica. No caso do composto estudado, todos os carbonos e prótons foram satisfatoriamente simulados, gerando modelos lineares (experimental $\mathrm{x}$ teórico) com graus de correlação estatística superiores a $97 \%$.

\section{AGRADECIMENTOS}

Os autores agradecem a UFPA e ao IFPA pela estrutura e apoio a pesquisa

\section{REFERÊNCIAS}

[1] Paranhos, F. L., Costa, De Albuquerque, A. C. F., Borges, R. M., Junior, F. M. S., Amorim M. B. High Cost-Effectiveness Ratio: GIAO-MPW1PW91/ 6-31G(d)//MPW1PW91/6-31G(d) Scaling Factor for 13C Nuclear Magnetic Resonance Chemical Shifts Calculation. Journal of Computational and Theoretical Nanoscience. 2014. Vol. 11, p. 1-7. ISSN: 1546-1955. DOI: 10.1166/jctn.2014.3341.

[2] Silva, S. O.; Peixoto, R. N. S.; Silva, J. R. A.; Alves, C. N.; Guilhon, G. M. S. P.; Santos, L. S.; Brasil, D. S. B. Identification of (-) (E)-N-[2(S)-Hydroxy-2-(4-hydroxyphenyl) ethyl]ferulamide, a Natural Product Isolated from Croton Pullei: Theoretical and Experimental Analysis. International Journal of Molecular Sciences. 2011, vol. 12, p. 9389-9403. ISSN 1422-0067. DOI:10.3390/ijms 12129389

[3] De Souza, F.S.; Silva, S.O.; Faria, L.J.L. Alves, C.N.; Muller, A.H., Guilhon, G.M.S.P.; Brasil, D.S.B. Dados Espectroscópicos de Diterpenos Labdânicos: Uma Análise Teórica via RMN e DFT. Química Nova. 2015, vol. 38, nº 5, p. 645-650. ISSN 01048899. DOI: http://dx.doi.org/10.5935/0100-4042.20150057

[4] Amador, D. H. T.; Silva, S. de O.; Deus, R. J. A.; Castro, K. C. F.; Docelli, M. D.; Martins, F. T.; Ellena, J.; Polikarpov, I.; Da silva, M. N.; Arruda, M. S. P.; Macedo, L. G.; Alves, C. N.; Brasil, D. do S. B.; Crystal Structure of Limonoid 6- $O$ Acetylswietephragmin $E$ and Theoretical Study of Nuclear Magnetic Resonance Spectra of Phragmalin Limonoids Advanced Science Letters. 2012, vol. 18, p. 150-157. ISSN: 1936-6612. DOI: https://doi.org/10.1166/asl.2012.4398

[5] Brasil, D. S. B.; Muller, A. H.; Guilhon, G. M. S. P.; Alves, C. N.; Peris, G.; Llusard, R.; Molinerd, V.; Isolation, X-ray Crystal Structure and Theoretical Calculations of the New Compound 8-epicordatin and Identification of Others Terpenes and Steroids from the Bark and Leaves of Croton palanostigma Klotzsch. Journal of The Brazilian Chemical Society. 2010, vol.21, $\mathrm{n}^{\circ}$ 4, p. 731-739. ISSN 0103-5053. DOI http://dx.doi.org/10.1590/S0103-50532010000400021

[6] Paranhos, F. L., Costa, De Albuquerque, A. C. F., Borges, R. M., Junior, F. M. S., Leitão, G.G., Amorim M. B. Bathysa australis Vanillic Acid (4-hydroxy-3-methoxybenzoic acid) Isolation by Countercurrent Chromatographic and Characterization by NMR $1 \mathrm{H}$ and $13 \mathrm{C}$ Experimental and Theoretical GIAO-B3PW91/cc-pVDZ//B3PW91/cc-pVDZ Chemical Shifts. Journal of Computational and Theoretical Nanoscience. 2014. Vol. 11, p. 1-6. ISSN: 1546-1955. DOI: $10.1166 /$ jctn.2014.3559

[7] Stewart, J.J.P. Optimization of parameters for semiempirical methods I. Method. Journal of Computational. Chemistry. 1989, vol. $10, \mathrm{n}^{\circ} 2$, p. 209-220. ISSN: 0192-8651 DOI: $10.1002 /$ jcc. 540100208 
[8] HyperChemTM Release $\mathbf{7 . 5}$ for Windows Molecular Modeling System. Hypercube: Gainesville, FL, 2002.

[9] Gaussian 09, Revision A.1, Frisch, M. J.; Trucks, G. W.; Schlegel, H. B.; Scuseria, G. E.; Robb, M. A.; Cheeseman, J. R.; Scalmani, G.; Barone, V.; Mennucci, B.; Petersson, G. A.; Nakatsuji, H.; Caricato, M.; Li, X.; Hratchian, H. P.; Izmaylov, A. F.; Bloino, J.; Zheng, G.; Sonnenberg, J. L.; Hada, M.; Ehara, M.; Toyota, K.; Fukuda, R.; Hasegawa, J.; Ishida, M.; Nakajima, T.; Honda, Y.; Kitao, O.; Nakai, H.; Vreven, T.; Montgomery, Jr., J. A.; Peralta, J. E.; Ogliaro, F.; Bearpark, M.; Heyd, J. J.; Brothers, E.; Kudin, K. N.; Staroverov, V. N.; Kobayashi, R.; Normand, J.; Raghavachari, K.; Rendell, A.; Burant, J. C.; Iyengar, S. S.; Tomasi, J.; Cossi, M.; Rega, N.; Millam, J. M.; Klene, M.; Knox, J. E.; Cross, J. B.; Bakken, V.; Adamo, C.; Jaramillo, J.; Gomperts, R.; Stratmann, R. E.; Yazyev, O.; Austin, A. J.; Cammi, R.; Pomelli, C.; Ochterski, J. W.; Martin, R. L.; Morokuma, K.; Zakrzewski, V. G.; Voth, G. A.; Salvador, P.; Dannenberg, J. J.; Dapprich, S.; Daniels, A. D.; Farkas, Ö.; Foresman, J. B.; Ortiz, J. V.; Cioslowski, J.; Fox, D. J. Gaussian, Inc., Wallingford CT, 2009.

[10] Miertš, S.; Scrocco, E.; Tomasi, J. Electrostatic interaction of a solute with a continuum. A direct utilizaion of $A B$ initio molecular potentials for the prevision of solvent effects. Chemical Physics. 1981, vol. 55, n. 1, p. 117-129. ISSN: 03010104. DOI: 10.1016/0301-0104(81)85090-2

[11] MIERTUŠ, S.; TOMASI, J. Approximate evaluations of the electrostatic free energy and internal energy changes in solution processes. Chemical Physics. 1982, vol. 65, n 2, p. 239 245. ISSN: 0301-0104. DOI: http://dx.doi.org/10.1016/0301-0104 (82)85072-6

[12] Minitab Release 14 for Window. Minitab Inc., State College, PA, 2003.

[13] Gaudio A. C, Zandonade E. Proposição, validação e análise dos modelos que correlacionam estrutura química e atividade biológica. Química Nova. 2001; vol. 24, p. 658-71. ISSN 01004042. DOI: http://dx.doi.org/10.1590/S0100-40422001000500013 